\title{
Grain boundary segregation, phase formation, and their influence on the coercivity of rapidly solidified $\mathrm{SmFe}_{11} \mathrm{Ti}$ hard magnetic alloys
}

\author{
Dhanalakshmi Palanisamy, ${ }^{1, *}$ Semih Ener $\odot,{ }^{2}$ Fernando Maccari, ${ }^{2}$ Lukas Schäfer,${ }^{2}$ Konstantin P. Skokov, ${ }^{2}$ \\ Oliver Gutfleisch, ${ }^{2}$ Dierk Raabe, ${ }^{1}$ and Baptiste Gault $0^{1,3}$ \\ ${ }^{1}$ Max-Planck-Institut für Eisenforschung GmbH, 40237 Düsseldorf, Germany \\ ${ }^{2}$ Functional Materials, Department of Material Science, Technische Universität Darmstadt, 64287 Darmstadt, Germany \\ ${ }^{3}$ Department of Materials, Royal School of Mines, Imperial College, Prince Consort Road, London SW7 2BP, United Kingdom
}

(Received 25 January 2020; revised manuscript received 23 March 2020; accepted 13 April 2020; published 8 May 2020)

\begin{abstract}
$\mathrm{SmFe}_{11}$ Ti-based alloys have potential as permanent magnet materials; however, until now, crystallographically textured bulk permanent magnets have not yet been produced from this alloy system. This is partly due to the lack of information on the morphology and composition of grain boundary phases present in the Fe-rich $\mathrm{Sm}-\mathrm{Fe}-\mathrm{Ti}$ alloys. Here we investigated the microstructure of a $\mathrm{Sm}_{1.25} \mathrm{Fe}_{11} \mathrm{Ti}$ alloy by using correlative transmission electron microscopy and atom-probe tomography, combined with magneto-optical Kerr effect (MOKE) probing to relate the material's micro- and nanostructure to its properties. The grains of the $\mathrm{Sm}(\mathrm{Fe}, \mathrm{Ti})_{12}$ matrix phase are separated by grain boundaries exhibiting a different composition over 3-4 nm width. They contain $>75$ at $\%$ of the ferromagnetic element $\mathrm{Fe}$, with an enrichment of $\mathrm{Sm}$ of up to $16.6 \mathrm{at} \%$ and a depletion in $\mathrm{Ti}$, down to approx. 3.4 at $\%$. We believe that the grain boundary is ferromagnetic at room temperature, which makes the magnetic decoupling of the grains practically impossible, which, in turn, leads to a low coercivity of $\mathrm{SmFe}_{11}$ Ti-based alloys. MOKE measurements reveal the strong ferromagnetic coupling across the grain boundary, causing the nucleation of reversal magnetic domains when exposed to low magnetic fields. In a triple-junction area we identified three other ferromagnetic phases: $\mathrm{Sm}_{3}(\mathrm{Fe}, \mathrm{Ti})_{29}, \mathrm{SmFe}_{2}$, and $\mathrm{Fe}_{2} \mathrm{Ti}$. These details bring out the scope of further adjustment of the coercivity in the Sm-Fe-Ti alloy system by grain boundary segregation engineering through the reduction of the presence of ferromagnetic phases to ensure a magnetic decoupling of the micrometer-sized $\mathrm{Sm}(\mathrm{Fe}, \mathrm{Ti})_{12}$ grains.
\end{abstract}

DOI: 10.1103/PhysRevMaterials.4.054404

\section{INTRODUCTION}

$\mathrm{RE}_{2} \mathrm{Fe}_{14} \mathrm{~B}$-based permanent magnets, where the main rareearth (RE) element is Nd with small additions of Pr, Dy, or Tb, exhibit outstanding magnetic properties with energy product values as high as $400 \mathrm{~kJ} / \mathrm{m}^{3}$ [1]. They find applications in a range of technologies such as household electronics and electrical appliances, robotics, electric vehicles, and many more [2,3]. Nevertheless, the low abundance of RE elements in the earth crust and the high demand has led to a supply crisis in 2011, and the cost of RE metals remains rather high. Hence, there are serious research efforts aimed either at reducing the amount of expensive RE (Tb,Dy) used for manufacturing these magnets or at searching for new RE-lean and RE-free hard magnetic materials with similar properties.

\footnotetext{
*Corresponding author: d.palanisamy@mpie.de
}

Published by the American Physical Society under the terms of the Creative Commons Attribution 4.0 International license. Further distribution of this work must maintain attribution to the author(s) and the published article's title, journal citation, and DOI. Open access publication funded by the Max Planck Society.
One of the suitable groups of low-RE-containing alloys is $\mathrm{REFe}_{12}$-based intermetallics with a $\mathrm{ThMn}_{12}$-type tetragonal crystal structure, i.e., space group I4/ $\mathrm{mmm}$ [4-7]. Among these intermetallics, $\mathrm{SmFe}_{12}$-based compounds are the most promising ones, since they exhibit large uniaxial magnetocrystalline anisotropy of up to $12 \mathrm{~T}$ at room temperature and large magnetization values $\left(\mu_{o} M_{S}\right)$ of up to $1.90 \mathrm{~T}$ with the lowest RE content [8,9]. These exceptional intrinsic values were achieved for $\mathrm{Sm}\left(\mathrm{Fe}_{1-x} \mathrm{Co}_{x}\right)_{12}$-based compounds $(x=0$, 0.1 , and 0.2 ) which were epitaxially grown as thin films to up to $\sim 500 \mathrm{~nm}$ thickness, in which the $\mathrm{ThMn}_{12}$ tetragonal structure had been stabilized due to the constraints on the crystal lattice imposed by a V buffer layer [9]. Indeed, $\mathrm{REFe}_{12}$ intermetallics with a $\mathrm{ThMn}_{12}$ structure are not stable in the bulk state, and therefore they require additional dopants for stabilization of the $1: 12$ phase, such as $\mathrm{V}, \mathrm{Mo}, \mathrm{Ti}, \mathrm{W}$, or $\mathrm{Cr}$ [5,10-14]. However, the addition of nonmagnetic dopants causes a dilution of the magnetization and reduces the Curie temperature. Thus, the amount of dopant to be added should be as low as possible. For $\mathrm{SmFe}_{12}$, Ti has been found to be very effective, with only approx. 7 at $\%$ required for stabilizing the $\mathrm{ThMn}_{12}$ structure [15].

In order to convert good intrinsic properties, i.e., spontaneous magnetization and magnetic anisotropy, into technically acceptable extrinsic properties, i.e., high remanent 
magnetization and coercivity, the relationship between the microstructure of $\mathrm{REFe}_{12}$-based alloys and their properties must be established. The microstructure optimization in $\mathrm{SmFe}_{11} \mathrm{Ti}$ bulk magnets, involving grain-size reduction and grain boundary engineering, has been extensively investigated in the past decades, and this challenging task is still far from being completed for this new class of alloys. Kuno et al. [16] recently showed comparable magnetization to the benchmark $\mathrm{Nd}_{2} \mathrm{Fe}_{14} \mathrm{~B}$ for $(\mathrm{Sm}, \mathrm{Zr})(\mathrm{Fe}, \mathrm{Co})_{11.5} \mathrm{Ti}_{0.5}$ produced by stripcasting, but at the same time, they reported no coercivity. They also described multiple phases in the alloy other than the $\mathrm{ThMn}_{12}$ type (the main phase will be referred to as $1: 12$ hereafter) $[17,18]$. The most detrimental phase identified for the low coercivity and remanence is the $\alpha$-(Fe,Co), which can be avoided by optimizing the composition and solidification rate. For example, strip-casting of a stoichiometric $\mathrm{SmFe}_{11} \mathrm{Ti}$ alloy led to complete suppression of $\alpha$-Fe [16]. Later, Tozman et al. [18] studied the influence of the actual volume fraction of the 1:12 phase on the intrinsic properties of the $(\mathrm{Sm}, \mathrm{Zr})(\mathrm{Fe}, \mathrm{Co})_{11.5} \mathrm{Ti}_{0.5}$ alloy. However, no detailed investigations were performed to identify the nature and origin of other phases that may be formed during synthesis and processing and likely influence the hard magnetic properties of these materials. In order to achieve a high coercivity, it is crucial to optimize the microstructure and interfacial regions between the main phase $\mathrm{SmFe}_{11} \mathrm{Ti}$ and the grain boundary phases. As a starting point, it is important to identify all additional phases present in alloys with $\mathrm{SmFe}_{11} \mathrm{Ti}$ phase as a matrix phase. Moreover, a detailed analysis of intergranular interfaces is indispensable to enable further microstructure optimization.

Here, by using correlative transmission electron microscopy (TEM) and atom-probe tomography (APT), we successfully identified the chemical composition and internal structure of grain boundaries formed during rapid solidification of the ternary $\mathrm{SmFe}_{11} \mathrm{Ti}$ alloy. Additionally, we employed Kerr microscopy to observe the effect of grain boundaries on the propagation of magnetic domain walls (DWs) in the alloy. We show evidence of Sm enrichment to grain boundaries and report the formation of the $\mathrm{Sm}_{3}(\mathrm{Fe}, \mathrm{Ti})_{29}$ (referred to as 3:29 below), $\mathrm{SmFe}_{2}$, and $\mathrm{Fe}_{2} \mathrm{Ti}$ Laves phases specifically on the grain boundaries. Our results will help rationalize the design of hard magnets based on the 1:12 alloy system.

\section{EXPERIMENTAL}

A polycrystalline $\mathrm{Sm}_{1.25} \mathrm{Fe}_{11} \mathrm{Ti}$ ingot was prepared by melting high-purity elemental constituents in an induction furnace under a purified argon gas atmosphere. The Sm-rich off-stoichiometric composition was used to compensate for the evaporation losses during sample preparation. By using an arc-melting setup, the ingot was suction-cast into rectangular slabs of $\sim 0.5 \mathrm{~mm}$ thickness to ensure rapid solidification and cooling. After suction casting, the composition was checked by using energy-dispersive $\mathrm{x}$-ray spectroscopy in the scanning electron microscope. The bulk composition of the ingot was $\mathrm{Sm}_{7.7} \mathrm{Fe}_{85.5} \mathrm{Ti}_{6.8}$, i.e., the stoichiometry of the material was closer to $\mathrm{SmFe}_{11.1} \mathrm{Ti}_{0.9}$, i.e., with a slight excess of Fe. Hence, hereafter, the composition of the alloy is referred to as $\mathrm{SmFe}_{11.1} \mathrm{Ti}_{0.9}$. The suction-cast slabs were wrapped in
Mo foil, sealed in quartz ampules under Ar atmosphere, and annealed at $1175^{\circ} \mathrm{C}$ for $30 \mathrm{~min}$ to homogenize the sample and stabilize the $\mathrm{Sm}(\mathrm{Fe}, \mathrm{Ti})_{12}(1: 12)$ phase.

The grains and grain boundary phases were located using backscattered-electron (BSE) imaging in a scanning electron microscope (SEM, FEI Helios 600i) operated at $30 \mathrm{kV}$ using a $1.4 \mathrm{nA}$ current. Correlative TEM and APT investigations were carried out using a JEOL 2200FS, aberration-corrected TEM (FEI TITAN) in scanning transmission electron microscopy (STEM) mode and a local-electrode atom probe (LEAP 5000 $\mathrm{XR}$, Cameca) equipped with a reflectron, which increases the time-of-flight path and hence the compositional sensitivity. For APT, needle-shaped specimens were prepared from the grain boundary regions using a dual-beam SEM/focused-ionbeam (FIB) instrument (FEI Helios Nanolab 600i) with an in situ, in-plane lift-out procedure detailed in Refs. [19-21]. The lamellae containing the grain boundary region were positioned and Pt-welded using the in situ gas-injection system on the electropolished posts of a halved TEM Mo grid. This Mo grid with the attached specimens was held in a correlative holder designed in-house as described in Ref. [22]. The specimens were sharpened by $\mathrm{Ga}$ ions accelerated at $30 \mathrm{kV}$ with currents ranging from $80 \mathrm{pA}$ to $0.78 \mathrm{nA}$ and then checked by TEM. This protocol was repeated until the grain boundary was located within $\sim 500 \mathrm{~nm}$ from the apex of the specimen. Before the APT analysis, the specimens were cleaned by $5 \mathrm{kV}$ with $8 \mathrm{pA}$ current to remove near-surface regions that were damaged by the high-energy Ga ions. Subnanometer compositional analysis was performed using the APT instrument operated in laser pulsing mode with a pulse repetition of $200 \mathrm{kHz}$ and a pulse energy of $40 \mathrm{pJ}$. The specimens were kept at a base temperature of $60 \mathrm{~K}$ with a target detection rate of five ions detected per 1000 pulses. The APT reconstruction and analysis were performed using IVAS 3.8.2 software.

The room-temperature isothermal magnetization measurements $[M(H)]$ were carried out using a Quantum Design physical properties measurement system (PPMS) equipped with a vibrating sample magnetometer (VSM). Magnetooptical Kerr effect (MOKE) measurements were carried out using a Zeiss Axio Imager.D2m polarized light microscope. The MOKE measurements were conducted at room temperature, and the external magnetic field was applied horizontally with a small electromagnet setup which can go up to $380 \mathrm{mT}$.

\section{RESULTS}

\section{A. Composition of grain boundaries in the $\mathrm{SmFe}_{11.1} \mathbf{T i}_{0.9}$ alloy}

Figure 1(a) shows an overview BSE micrograph containing four different microstructural features marked as 1, 2, 3, and 4, respectively, corresponding to grain boundaries, and three phases (2, 3, and 4) appearing with different electron backscattering contrast. We also observe that the three phases were mostly found at the grain boundaries. Next we will analyze the structure, chemistry, and topology of these features.

An APT specimen was prepared from a grain boundary region with an orientation as shown by the dashed red triangle in Fig. 1(a). Figure 1(b) shows a brightfield (BF) TEM image of the APT specimen with clear diffraction contrast 
(a)

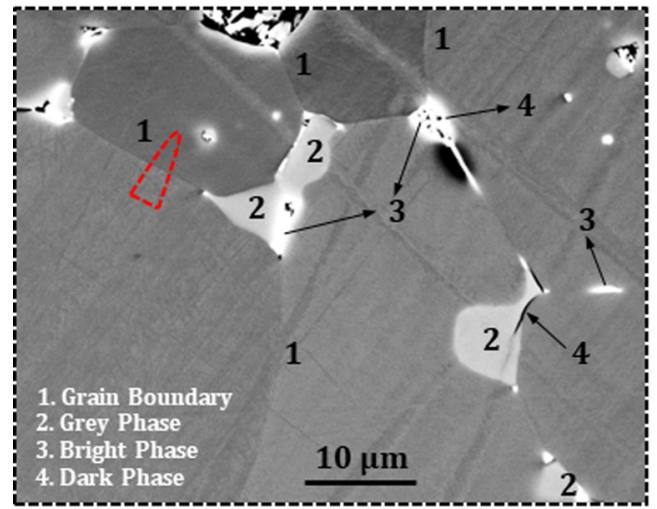

(c)

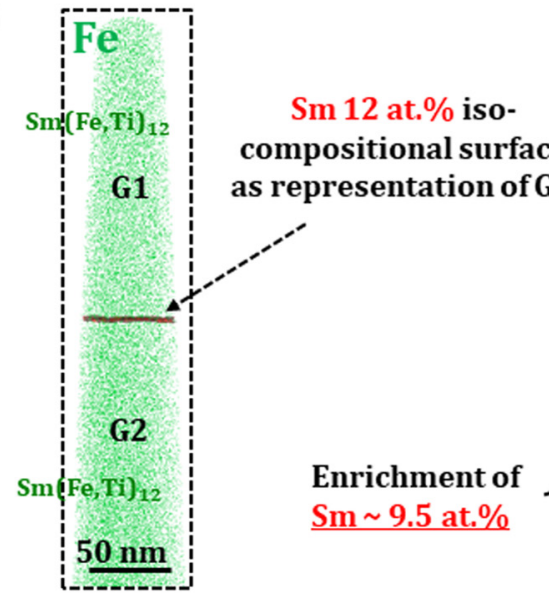

(b)

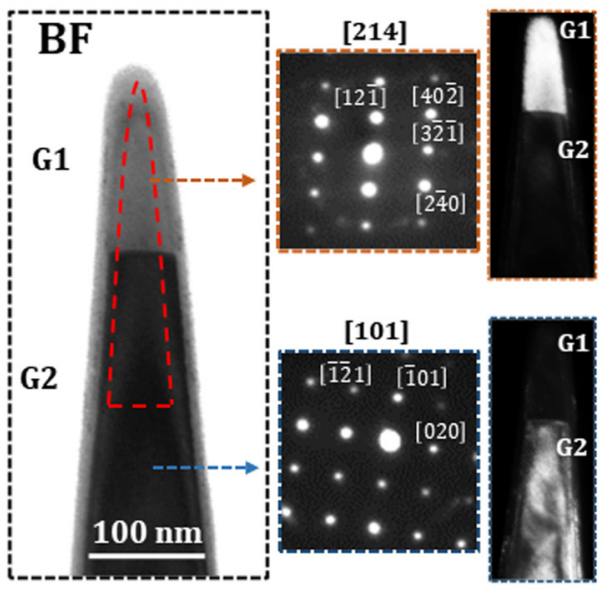

(d) Compositional profile across GB

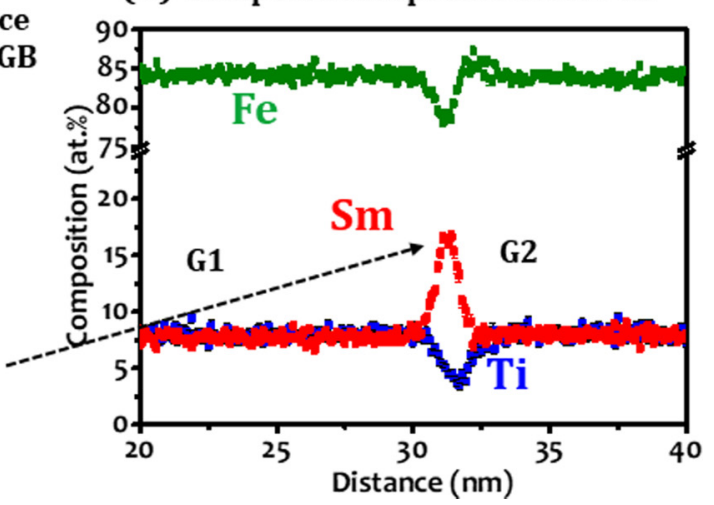

FIG. 1. (a) An overview BSE SEM image for the rapidly solidified and annealed $\mathrm{SmFe}_{11.1} \mathrm{Ti}_{0.9}$ alloy highlighting four different features comprising grain boundaries and three different phases, revealed by contrast differences, identified as 1, 2, 3, and 4, respectively. (b) A BF TEM image of an APT specimen showing two grains (G1 and G2) separated by a grain boundary with their respective diffraction patterns (DPs) and darkfield (DF) images. (c) APT reconstruction with distribution of Fe atoms (green color), Sm 12 at\% iso-compositional surface representing the grain boundary. (d) One-dimensional compositional profiles across the Sm 12 at\% iso-compositional surface. BSE: backscatter electrons; BF: brightfield; APT: atom-probe tomography; GB: grain boundary.

between the two grains (G1 and G2) separated by a grain boundary. The diffraction patterns (DPs) taken from G1 and G2 along a certain zone axes are also shown. Both DPs were indexed as the $\mathrm{Sm}(\mathrm{Fe}, \mathrm{Ti})_{12}$ phase (1:12) taken along the [214] zone axes for G1 and [101] for G2, with a space group of $I 4 / \mathrm{mmm}$ and lattice parameters $a=b=0.8589 \mathrm{~nm}$ and $c=0.4807 \mathrm{~nm}$. The darkfield (DF) images taken from the [12î] and [12î1] diffraction spots from the respective zones highlight the corresponding grains.

The APT specimen was then analyzed to obtain the composition of the grain boundary and the adjacent grains. Figure 1(c) shows the APT reconstruction with the distribution of $\mathrm{Fe}$ (green) atoms and an iso-composition surface (in red) with a threshold of 12 at $\% \mathrm{Sm}$, revealing the position of the grain boundary. Figure 1(d) shows the one-dimensional composition profile across the grain boundary with an enrichment of up to $16.6 \pm 0.26$ at $\% \mathrm{Sm}$, while $\mathrm{Fe}$ and $\mathrm{Ti}$ are depleted relative to the composition of the adjacent grains. It is important to note that the grain boundary composition contains up to $78.9 \pm 0.5$ at $\% \mathrm{Fe}$, i.e., a strongly ferromagnetic element. The composition of the two grains was measured to be $\mathrm{Sm}_{7.7 \pm 0.4} \mathrm{Fe}_{84.4 \pm 0.6} \mathrm{Ti}_{7.9 \pm 0.4}$, matching the composition of the $\mathrm{Sm}(\mathrm{Fe}, \mathrm{Ti})_{12}$ phase, i.e., of the $1: 12$ phase, as also confirmed from the diffraction analysis.

\section{B. Structure and composition of $\mathrm{Sm}_{3}(\mathrm{Fe}, \mathrm{Ti})_{29}$ phase in the $\mathrm{SmFe}_{11.1} \mathbf{T i}_{0.9}$ alloy}

Figure 2(a) shows a BF image of an APT specimen taken from a region of the sample containing the phase denoted as 2 [see Fig. 1(a)]. We observe a distinct diffraction contrast between the different phases within the specimen. Figures 2(b) and 2(c) show the DPs and DF images from the specimen. The DP from the top part of the specimen is indexed as a [214] zone axis pattern from the 1:12 matrix, while the DP from the middle part of the specimen is indexed as a [122] zone axis pattern from the $\mathrm{Sm}_{3}(\mathrm{Fe}, \mathrm{Ti})_{29}$ (3:29) monoclinic phase with a space group of $P 2_{1} / c$ and lattice parameters of $a=1.063 \mathrm{~nm}, b=0.857 \mathrm{~nm}, c=0.972 \mathrm{~nm}$, and $\beta=97^{\circ}$. Figure 2(d) shows the APT reconstruction of the same specimen with the distribution of $\mathrm{Fe}$ (green) atoms and a $\mathrm{Ti} 7$ at $\%$ iso-composition surface (in blue) delineating the phase boundary. The composition profile across the phase boundary is shown in Fig. 2(e). The phase composition is 
(a)

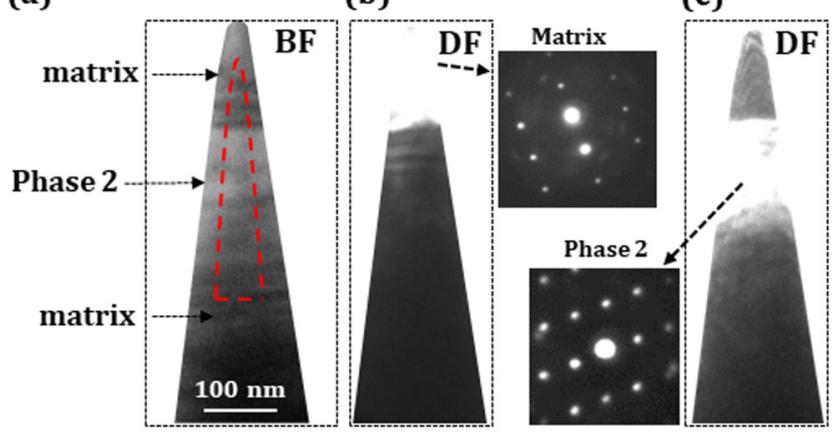

(d)

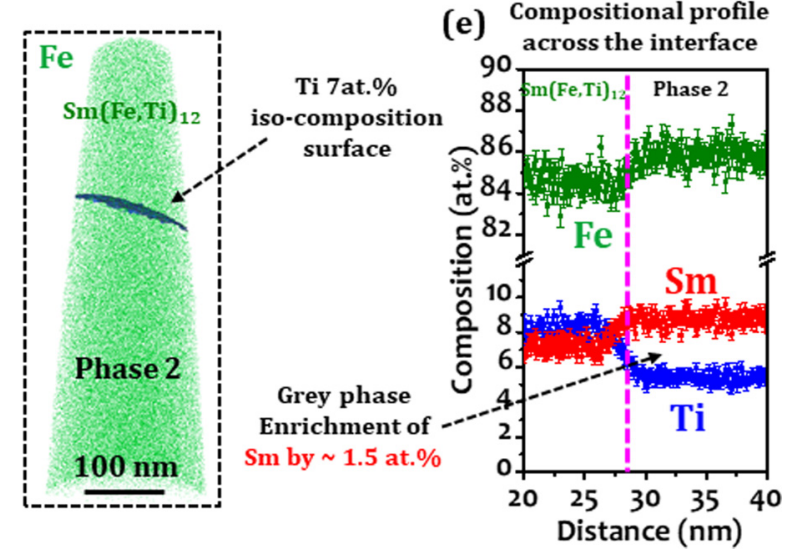

FIG. 2. (a) A BF TEM image of an APT specimen showing two different contrast regions with their respective diffraction patterns (DPs) and (b), (c) darkfield (DF) images. (d) APT reconstruction with distribution of $\mathrm{Fe}$ atoms (green color) and $\mathrm{Ti} 7$ at $\%$ isocompositional surface representing the phase boundary. (e) $1 \mathrm{D}$ compositional profiles across the Ti 7 at\% iso-compositional surface. BF: brightfield; APT: atom-probe tomography.

$\mathrm{Sm}_{8.8 \pm 0.4} \mathrm{Fe}_{85.8 \pm 0.5} \mathrm{Ti}_{5.4 \pm 0.3}$, which is close to the composition of the $\mathrm{Sm}_{3}(\mathrm{Fe}, \mathrm{Ti})_{29}$ phase, i.e., the 3:29 phase has here been confirmed by the diffraction analysis.

\section{Structure and composition of the $\mathrm{SmFe}_{2}$ and $\mathrm{Fe}_{2} \mathrm{Ti}$ phases in the $\mathrm{SmFe}_{11.1} \mathbf{T i}_{0.9}$ alloy}

To analyze the compositions and structures of the phases marked 3 (bright) and 4 (dark), a TEM lamella was prepared from a region shown in Fig. 3(a), where the microstructure resembles that of a eutectic mixture. A BF image taken from the lamella is shown in Fig. 3(b). We observe two regions of different contrast as the lamella was oriented into a zone axis with respect to the bottom part. The diffraction pattern from the bottom region could be successfully indexed according to the 1:12 matrix along the [100] zone axis. No specific common orientation between the two regions could be identified, i.e., there is likely no specific orientation relationship between the phases. The DP taken from the bright phase has been indexed as $\mathrm{SmFe}_{2}$ Laves (C15) phase with a cubic crystal structure, probed along the [001] zone axis, with space group $F d \overline{3} m$ and lattice parameters $a=b=c=0.74164 \mathrm{~nm}$.

An APT specimen was prepared from the eutectic type region in the inclined volume marked by a red triangle in Fig. 3(a). The corresponding APT reconstruction [Fig. 3(d)] contains two phases and the phase boundary is highlighted by a Fe 77 at\% iso-composition surface. The composition profile across the surface reaches from the 1:12 matrix phase stoichiometry into a Sm-enriched phase with no Ti partitioning [Fig. 3(e)]. The composition of the Sm-enriched phase has been identified as $\mathrm{Sm}_{33.2 \pm 0.5} \mathrm{Fe}_{66.8 \pm 0.4}$, matching the $\mathrm{SmFe}_{2}$ stoichiometry, as also confirmed by the diffraction analysis.

Z-contrast high-angle-annular-darkfield scanning-TEM (HAADF STEM) imaging, shown in Fig. 4(b) taken from the region indicated by a white dashed square in Fig. 4(a), reveals the presence of regions with three different compositions. Figure 4(c) shows energy-dispersive spectroscopy (EDS) mapping from the same region, confirming that the contrast directly relates to the Sm composition in the three phases. The composition of the darker phase, which appears with a lower volume fraction, was not detected by APT and was measured by EDS to be $\left(\mathrm{Sm}_{4.9} \mathrm{Fe}_{68.9}\right) \mathrm{Ti}_{26.2}$. From the Fe-Ti phase diagram [23], this composition falls into the phase field of the $\mathrm{Fe}_{2} \mathrm{Ti}$ Laves (C14) phase that has a hexagonal crystal structure and space group $P 6_{3} / \mathrm{mmc}$.

Since in the present sample the Ti-rich dark regions appear as thin regions $(<50 \mathrm{~nm}$ thickness), we performed high-resolution STEM analysis for structural analysis as shown in Fig. 4(d). The sample was tilted to the [100] zone axis of the matrix phase and the image was taken centered on a Ti-rich dark region [brown checked box in Fig. 4(b)]. HR-STEM analysis reveals that even the Tirich phase is oriented in a specific zone along the [100] matrix zone axis. The fast-Fourier-transform (FFT) pattern from the Ti-rich dark region is also shown and has been indexed as the $\mathrm{Fe}_{2} \mathrm{Ti}$ hexagonal phase along the [1010] axis. This result shows that the matrix 1:12 phase and the $\mathrm{Fe}_{2} \mathrm{Ti}$ phase have an orientation relationship that can be expressed as $[101]_{1: 12} / /[10 \overline{1} 0]_{\mathrm{Fe}_{2} \mathrm{Ti}} / /(020)_{1: 12} / /(0002)_{\mathrm{Fe}_{2} \mathrm{Ti}}$. The Sm-rich $\mathrm{SmFe}_{2}$ phase does not exhibit a specific orientation relationship with the 1:12 matrix, as confirmed by the corresponding FFT performed within the checked red square.

\section{Room-temperature magnetic hysteresis and MOKE measurements for the $\mathrm{SmFe}_{11.1} \mathrm{Ti}_{0.9}$ alloy}

The room-temperature $M(H)$ magnetic characterization conducted on the $\mathrm{SmFe}_{11.1} \mathrm{Ti}_{0.9}$ sample is shown in Fig. 5. The measured coercivity for the sample is $0.04 \mathrm{~T}(0.4 \mathrm{kOe})$, far from what would be required to qualify it as a permanent magnet. For comparison, $\mathrm{SmFe}_{11} \mathrm{Ti}$ single-phase alloy powders with an average grain size of $\sim 50 \mu \mathrm{m}$ were prepared by ball milling. The coercivity of the magnetically decoupled $\mathrm{SmFe}_{11} \mathrm{Ti}$ powders is $\mathrm{Hc} \sim 0.1 \mathrm{~T}$, about twice the coercivity of the suction-cast $\mathrm{SmFe}_{11.1} \mathrm{Ti}_{0.9}$ sample with a grain size of 80-150 $\mu \mathrm{m}$. Hence, MOKE measurements were carried to determine if the grain boundaries are magnetically coupled in the suction-cast $\mathrm{SmFe}_{11.1} \mathrm{Ti}_{0.9}$ sample.

The room-temperature MOKE images recorded from the same region of the sample are shown in Fig. 6(a) after application of external magnetic fields of $-15,0,150$, and $300 \mathrm{mT}$. The field was applied in the horizontal direction of the images in both positive (right-to-left) and negative (leftto-right) directions. Magnetic domains appear in the form of alternating regions of dark and light contrast. The orientation 
(a)
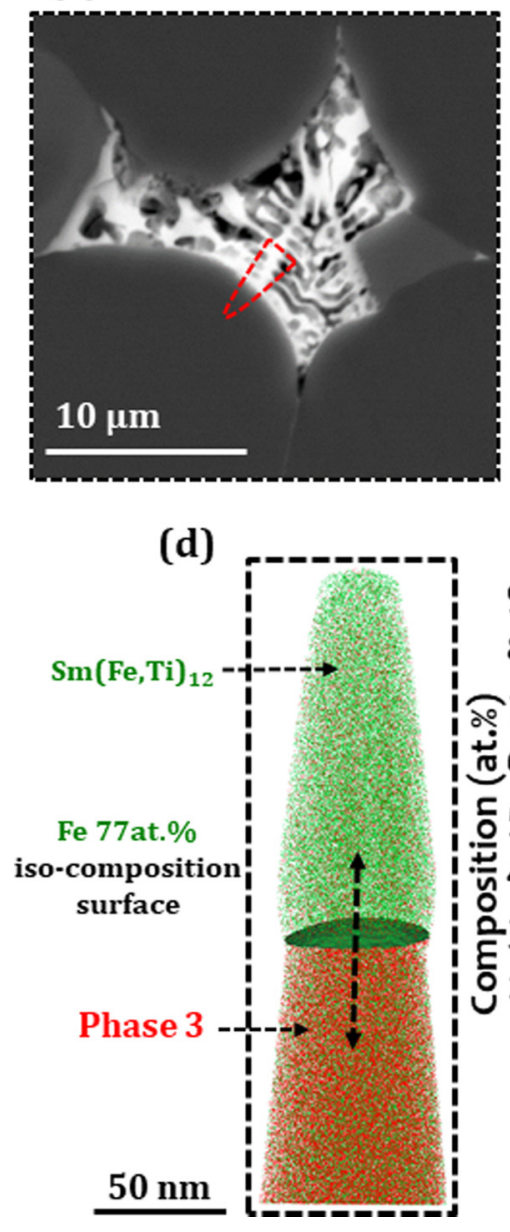

(b)

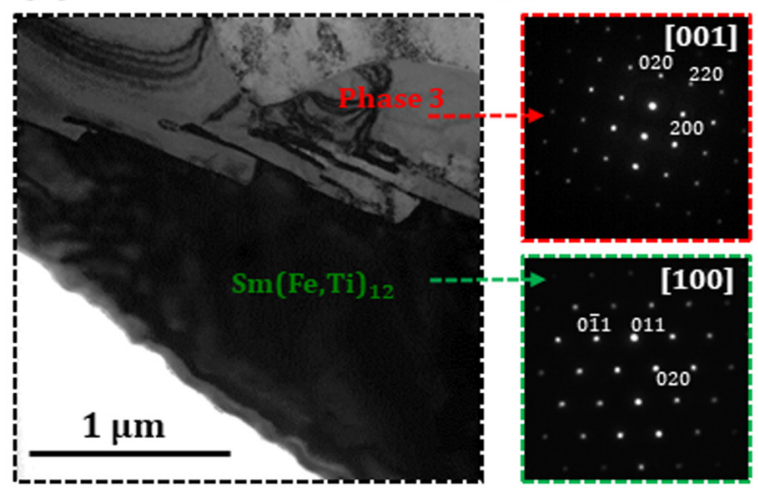

(e) Compositional profile across the interface

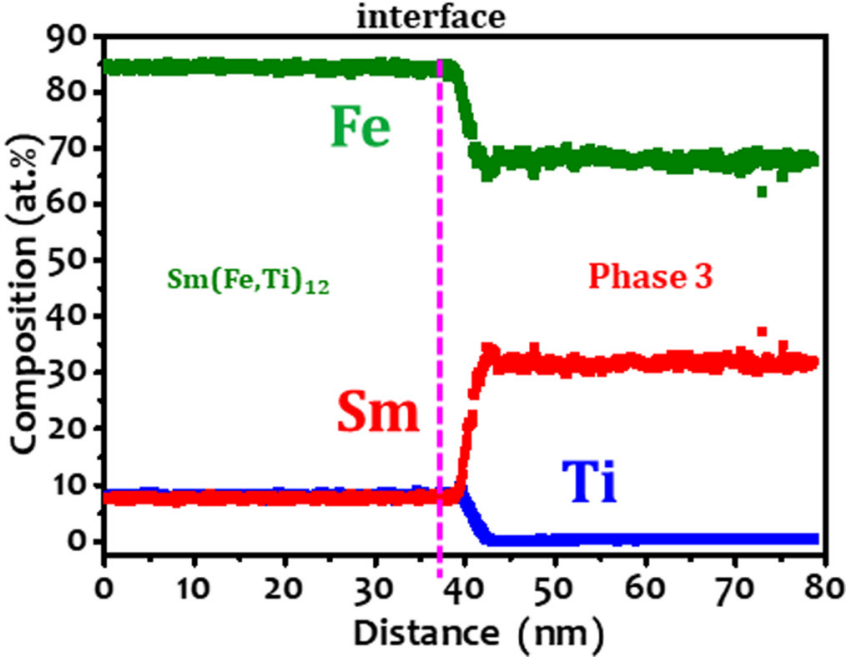

FIG. 3. (a) A BSE SEM image from a grain boundary eutectic region. (b) A BF TEM image of a lamella showing two different contrast regions with their respective (c) diffraction patterns (DPs). (d) APT reconstruction with distribution of Fe (green color) and Sm (red color) atoms and $\mathrm{Fe} 77$ at\% iso-compositional surface representing the phase boundary. (e) 1D compositional profiles across the Fe 77 at\% isocomposition surface.

of the domains depends on the crystallographic orientation of each individual grain. A strong magnetic coupling is visible between neighboring grains marked as 1, 2, 3, and 4 . We also provide in Fig. 6(b) a series of closeups on the region delineated by a dashed rectangle containing a grain boundary, indicated by a white ellipse, between grains 1 and 2 . For 0 , 150 , and $300 \mathrm{mT}$, we observe that the magnetic domains on the either side of the grain boundary have continuous light gray and dark gray contrasts, respectively, for grain 1 and for grain 2 . In the case of $-15 \mathrm{mT}$, a coupled switching of the magnetic domains is observed, with a change of the light gray and dark gray contrast in the vicinity of the grain boundary within grains 1 and 2 [see top image in Fig. 6(b)]. The change of the magnetic domains even under very small negative magnetic fields confirms the lack of magnetic isolation, which supports the strong magnetic coupling of the neighboring grains (grains 1 and 2).

At another grain boundary, between grains 3 and 4, which is indicated by a white arrow, the striplike domains of grain 3 and mazelike domains of grain 4 are magnetically coupled at the grain boundary. Significant changes in the magnetic domains in grain 3 and coupled changes in grain 4 are observed due to the applied magnetic field (see Supplemental Material animated GIF in Ref. [24]). Because of the high magnetocrystalline anisotropy of the phase, no significant changes in the domain pattern inside grain 4 are expected, yet due to the coupling through the grain boundary, minor changes in the domain pattern are observed. The mapping of the magnetic substructures revealed by MOKE shows magnetic features at the surface of the grains, which will generally show lower coercivity in comparison to the bulk beneath [25,26]. Yet the main character of the magnetic interaction among adjacent grains will not fundamentally change for either the surface or the bulk.

\section{DISCUSSION}

Similar to the microstructures reported for other $\mathrm{ThMn}_{12}$ type systems [27-30], for the $\mathrm{SmFe}_{11.1} \mathrm{Ti}_{0.9}$ system studied here, we expected to observe a nucleation-type coercivity mechanism. This mechanism implies that new magnetic domains can nucleate in the direction opposite to the magnetization within adjacent domains. This nucleation takes place at specific sites at the surface of the grains. This effect can significantly decrease the critical nucleation energy. 
(a) BF TEM image (b) HAADF STEM image
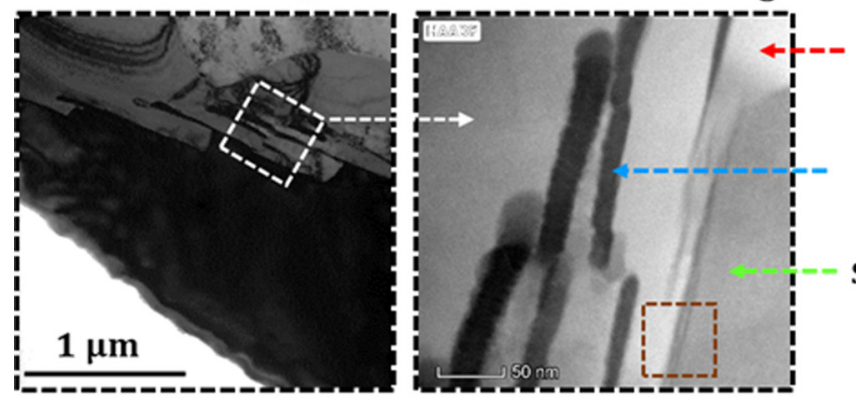

(c)

EDS elemental mapping
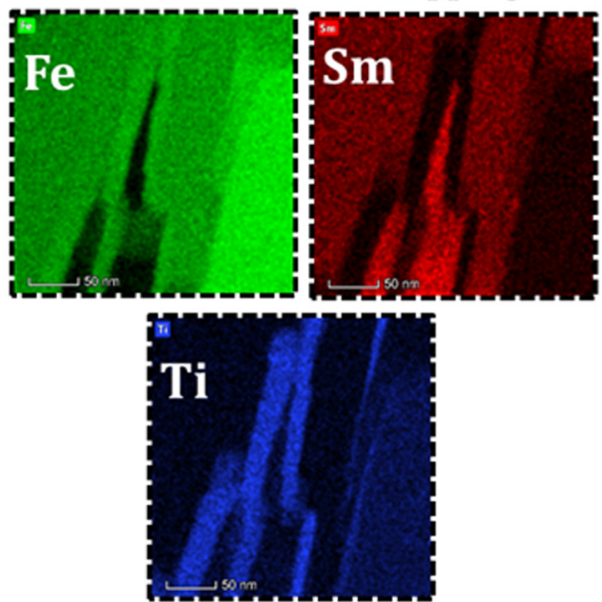

(d) High resolution HAADF STEM imaging

$\mathrm{SmFe}_{2}$

Phase 4

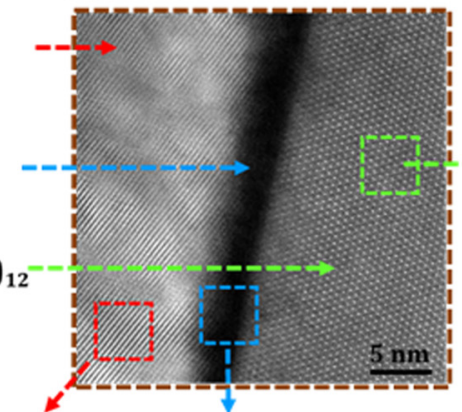

Phase 4
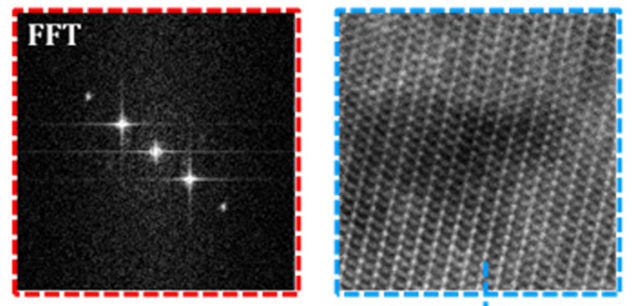

$\mathrm{Sm}(\mathrm{Fe}, \mathrm{Ti})_{12}$

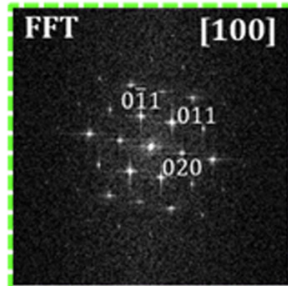

FIG. 4. (a) BF TEM image of the lamella showing the region of interest for HAADF STEM analysis (white checked rectangle). (b) HAADF STEM image with three different contrast features, and (c) EDS elemental mapping from the same region. (d) High-resolution HAADF STEM analysis on the region centered on the dark phase [brown dashed rectangle in Fig. 5(b)] with respective phase images and their FFTs. HAADF: High-angle annular darkfield imaging; STEM: scanning transmission electron microscopy. FFT: fast-Fourier-transform; EDS: energy-dispersive $\mathrm{x}$-ray spectroscopy.

Magnetic interactions among neighboring grains also reduce the bulk material's coercivity due to an increase in the local (effective) demagnetization field. Hence the magnetically

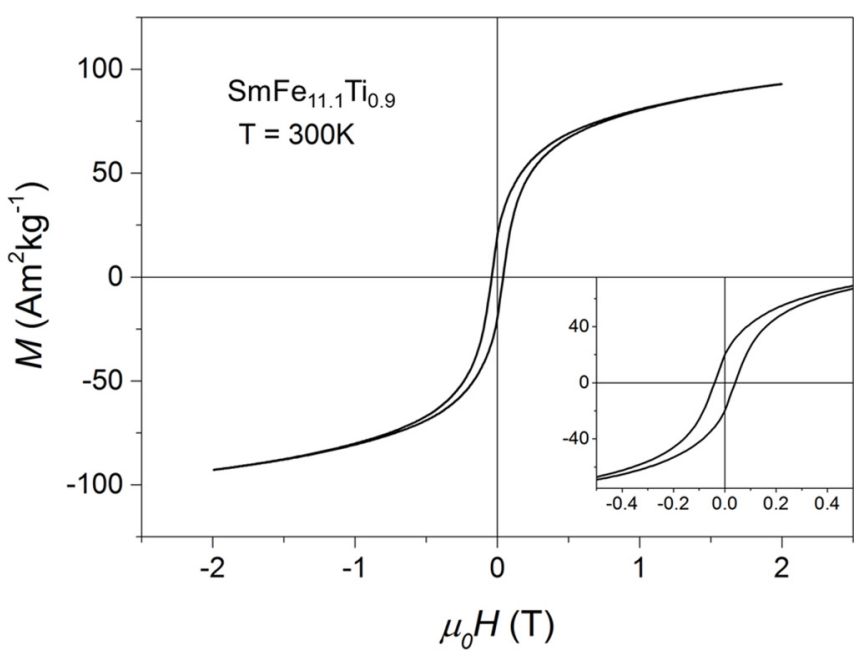

FIG. 5. Room-temperature magnetic hysteresis measurements of $\mathrm{SmFe}_{11.1} \mathrm{Ti}_{0.9}$ sample. Inset shows the low external field region for clarity. optimal microstructure of Sm-Fe-Ti nucleation-type magnets should consist of $\mathrm{SmFe}_{11} \mathrm{Ti}$ grains separated by thin paramagnetic grain boundaries, which could magnetically decouple the matrix grains. The magnetic domain structure mapping conducted in this study can thus provide insights into the local structure-chemistry-magnetism relations and provides some information on the degree of the magnetic decoupling. In an ideal permanent magnet, the domain patterns of the grains should be relatively independent, showing no correlation with the magnetic domain structure in the neighboring grains.

One of the most important processing guidelines to enhance the coercivity of a magnetic alloy is to refine its grain size up to the range of its critical single-domain size [31-33]. Several routes, such as mechanical alloying or rapid solidification, can be employed for grain refinement [34-36]. In the Sm-Fe-Ti alloy system, these techniques, in combination with additional alloying, were systematically employed to increase the coercivity [37-40]. The highest value achieved to date is only approx. $0.77 \mathrm{~T}(7.7 \mathrm{kOe})$ for a $\mathrm{Sm}_{1} \mathrm{Fe}_{8} \mathrm{Ti}_{0.75} \mathrm{~B}_{0.25}$ alloy processed through rapid solidification via melt-spinning [36], which resulted in a microstructure of 1:12 phase grains with grain sizes in the range of $20-50 \mathrm{~nm}$. Boron addition resulted in a reduced amount of $\alpha$-Fe. Even though having very fine grain sizes, the low coercivity value might be due to the 
(a)
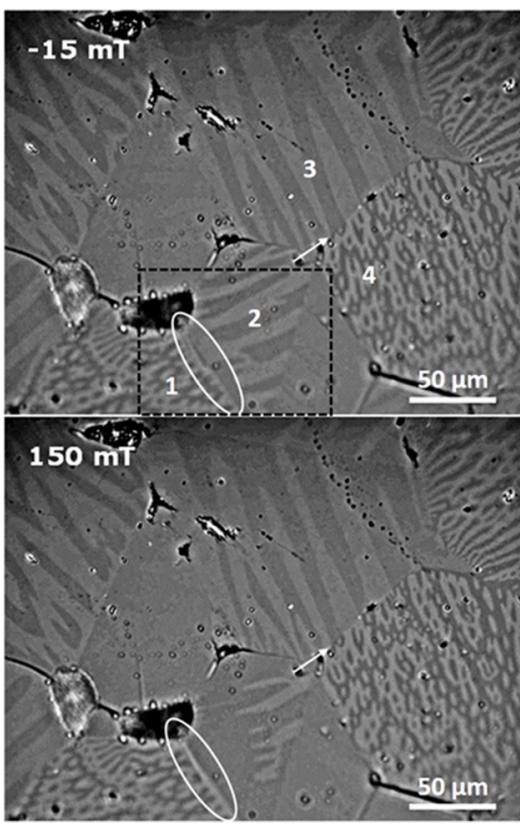
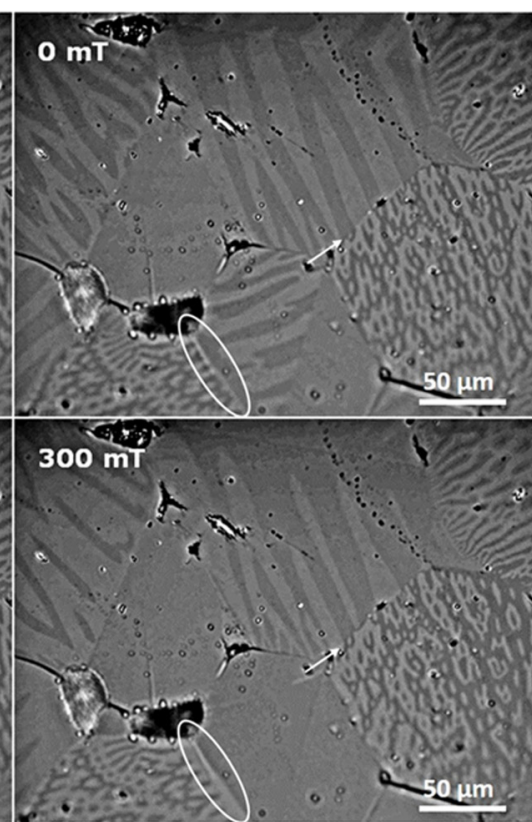

(b)

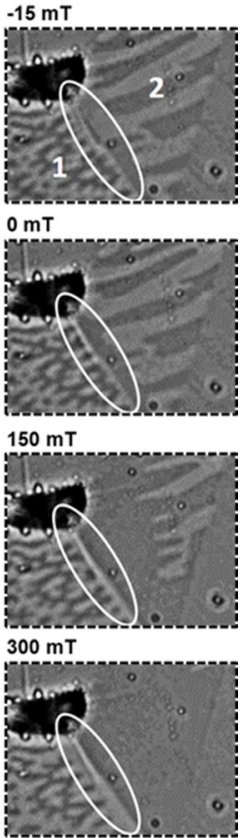

FIG. 6. Room-temperature magneto-optical Kerr microscopy measurements of the $\mathrm{SmFe}_{11.1} \mathrm{Ti}_{0.9}$ sample under different external magnetic fields. (a) Kerr images recorded from the same region of the microstructure after exposure to magnetic fields of $-15,0,150$, and $300 \mathrm{mT}$. (b) A series of closeup images from the region marked as a black dashed rectangle in (a) focused on the grain boundary between 1 and 2 . The magnetic field is applied along the horizontal axis from right-to-left direction.

exchange-coupled nanocrystalline grains. The major difficulty in using exchange-coupled magnets consists in the practical impossibility of aligning these grains in a magnetic field of reasonable magnitude (in industry a field of $1-2 \mathrm{~T}$ is commonly used). If single-domain nanocrystalline grains possess very pronounced shape anisotropy, by using hot compaction and hot deformation, it is possible to provide a high degree of texture in fully dense samples, e.g., die-upset NdFeB-based magnets. Unfortunately, the $\mathrm{SmFe}_{11.1} \mathrm{Ti}_{0.9}$ nanograins do not have a pronounced shape anisotropy, and all nanocrystalline $\mathrm{SmFe}_{11} \mathrm{Ti}$ magnets are bound to be isotropic. Thus, to obtain a texture in 1:12 magnets, it is necessary to employ traditional sintering of a green compact consisting of $2-10 \mu \mathrm{m}$ grains aligned in a magnetic field.

Ideally, an alloy intended for production of sintered permanent magnets should hence contain at least $90 \%$ of a highly anisotropic matrix phase, as well as a low-meltingpoint paramagnetic phase that should surround the grains to ensure good magnetic decoupling between adjacent grains. Here we have shown that one of the grain boundary phases is the 3:29 phase. This phase is ferromagnetic and has a pronounced uniaxial anisotropy $\left(H_{a}=3.4 T\right)$, i.e., lower than that of the surrounding 1:12 matrix phase [41,42]. Two other cubic phases, $\mathrm{SmFe}_{2}$ and $\mathrm{Fe}_{2} \mathrm{Ti}$, are also ferromagnetic with very low anisotropy fields [43-46]. The excess $\mathrm{Fe}$ and $\mathrm{Sm}$ in the alloy might also favor the formation of the $\mathrm{SmFe}_{2}$ phase. Hence, the presence of these phases is expected to contribute to the lowering of the overall coercivity of the bulk alloy. Yet, all materials studied earlier were synthesized with Sm-rich off-stoichiometric compositions, which likely lead to the formation of $\mathrm{Sm}$ - and Fe-rich grain boundary phases that result in a low coercivity.
We also reveal that, in addition to changes in the crystallography across the grain boundaries, the composition of the grain boundary can also be a major contributor for influencing the overall coercivity of the $\mathrm{SmFe}_{11.1} \mathrm{Ti}_{0.9}$ alloy. In a Nd-Fe-B-based alloy, also exhibiting the $\mathrm{ThMn}_{12}$ structure, the grain boundary composition was shown to directly influence the coercivity. More specifically, in fine grain alloy ( $\sim 50$ to $300 \mathrm{~nm})$, processed by hydrogen disproportionation desorption recombination (HDDR), the grain boundaries contain up to $\sim 70$ at $\%$ of strongly ferromagnetic elements $\mathrm{Fe}$ and Co [47]. The $\mathrm{Fe}+\mathrm{Co}$ composition is higher compared to the grain boundary composition in the sintered magnets $(\sim 60$ at $\%)$. As a result, a lower coercivity is observed for the HDDR-processed alloy $(\sim 1-1.5 \mathrm{~T})$, despite finer grains, than for the sintered alloy $(\sim 2 \mathrm{~T})[1,47]$. This high coercivity was attributed to the reduction in ferromagnetic elements in the composition of grain boundaries that leads to a reduction in the local magnetization. Furthermore, modifying the grain boundary composition by increasing the $\mathrm{Nd}$ concentration in the grain boundaries, i.e., lowering the ferromagnetic elements, was found to result in an increase in the coercivity of HDDR processed and hot deformed Nd-Fe-B magnets to 1.9 and $1.8 \mathrm{~T}$, respectively $[47,48]$. Micromagnetic simulations confirmed that domain-wall nucleation starts at much lower values of magnetic field for a ferromagnetic grain boundary compared to a nonmagnetic grain boundary [48]. In contrast, the presence of a nonmagnetic grain boundary can hinder the movement of reversed domains to the adjacent grains, which contributes to achieving relatively high coercivity. In the $\mathrm{SmFe}_{11.1} \mathrm{Ti}_{0.9}$ alloy studied herein, the presence of the strong ferromagnetic element $\mathrm{Fe}(>75$ at $\%)$ at the grain boundaries is expected to result in a similar effect of strong magnetic 
coupling between the 1:12 phase grains. Hence the grain boundaries are unable to either avoid nucleation or pin down the movement of domain walls.

Our results will guide further optimization of the alloy magnetic properties, i.e., the observed link between the composition of grain boundaries and the ferromagnetic coupling in-between the grains opens a window of opportunity for tuning the coercivity, similar to the approach used, for instance, for Nd-Fe-B-based magnets, by reducing the Fe composition at the grain boundaries. A more recent study on $\left(\mathrm{Sm}_{1.3} \mathrm{Zr}_{0.27}\right)\left(\mathrm{Fe}_{0.8} \mathrm{Co}_{0.2}\right)_{12}$ epitaxially grown thin films on $\mathrm{MgO}$ (001) single-crystal substrate revealed Sm-rich regions (Fe $\sim 62$ at $\%$ ) that are proposed to be the reason for promising intrinsic magnetic properties [49].

\section{CONCLUSIONS}

In conclusion, the microstructure of suction-cast and annealed $\mathrm{SmFe}_{11.1} \mathrm{Ti}_{0.9}$ magnetic alloy was investigated. APT revealed a distinct composition of grain boundaries compared to the adjoining 1:12 matrix phase grains. The grain boundaries contain more than 75 at $\% \mathrm{Fe}$. Additionally, three different phases, namely, monoclinic $\mathrm{Sm}_{3}(\mathrm{Fe}, \mathrm{Ti})_{29}$, cubic $\mathrm{SmFe}_{2}$, and $\mathrm{Fe}_{2} \mathrm{Ti}$ Laves phases, all form preferably at the grain boundaries and at triple junctions. MOKE measurements reveal a strong ferromagnetic coupling of the 1:12 grains across the grain boundaries. Based on these results, we assume that the grain boundaries, with more than 75 at $\%$ of ferromagnetic elements, behave like ferromagnetic regions and therefore no magnetic isolation is observed at the grain boundaries. These insights can be used as guidelines towards proper choices of alloying and processing conditions in order to obtain a finer grain-size distribution and a reduction of the $\mathrm{Fe}$ composition at grain boundaries, which should help improve the coercivity and allow for higher performance.

\section{ACKNOWLEDGMENTS}

The authors are grateful to U. Tezins, C. Broß, and A. Sturm for their technical support of the atom-probe tomography and focused-ion-beam facilities at the Max-PlanckInstitut für Eisenforschung. All authors from TU Darmstadt acknowledge financial support from the future pioneering program development of magnetic material technology for high-efficiency motors (MagHEM) commissioned by the New Energy and Industrial Technology Development Organization (NEDO). This work was also supported by the Deutsche Forschungsgemeinschaft (DFG, German Research Foundation), Project ID No. 405553726, TRR 270.
[1] K. Hono and H. Sepehri-Amin, Scr. Mater. 67, 530 (2012).

[2] K. P. Skokov and O. Gutfleisch, Scr. Mater. 154, 289 (2018).

[3] O. Gutfleisch, M. A. Willard, E. Brück, C. H. Chen, S. G. Sankar, and J. P. Liu, Adv. Mater. 23, 821 (2011).

[4] K. H. J. Buschow, J. Appl. Phys. 63, 3130 (1988).

[5] K. H. J. Buschow, J. Magn. Magn. Mater. 100, 79 (1991).

[6] Y. C. Yang, G. J. Long, B. Kebe, W. J. James, and J. Deportes, in The Rare Earths in Modern Science and Technology, edited by G. J. McCarthy, H. B. Silber, J. J. Rhyne, and F. M. Kalina (Springer US, Boston, MA, 1982), Vol. 3, pp. 403-406.

[7] G. C. Hadjipanayis, A. M. Gabay, A. M. Schönhöbel, A. Martín-Cid, J. M. Barandiaran, and D. Niarchos, Engineering 6, 141 (2020).

[8] D. Ogawa, T. Yoshioka, X. D. Xu, Y. K. Takahashi, H. Tsuchiura, T. Ohkubo, S. Hirosawa, and K. Hono, J. Magn. Magn. Mater. 497, 165965 (2020).

[9] Y. Hirayama, Y. K. Takahashi, S. Hirosawa, and K. Hono, Scr. Mater. 138, 62 (2017).

[10] M. Hagiwara, N. Sanada, and S. Sakurada, AIP Adv. 9, 035036 (2019).

[11] K. Ohashi, T. Yokoyama, R. Osugi, and Y. Tawara, IEEE Trans. Magn. 23, 3101 (1987).

[12] S. Sugimoto, T. Shimono, H. Nakamura, T. Kagotani, M. Okada, and M. Homma, Mater. Chem. Phys. 42, 298 (1995).

[13] X. C. Kou, E. H. C. P. Sinnecker, R. Grössinger, G. Wiesinger, T. Zhao, J. P. Liu, and F. R. de Boer, J. Magn. Magn. Mater. 140, 1025 (1995).

[14] I. Dirba, Y. Harashima, H. Sepehri-Amin, T. Ohkubo, T. Miyake, S. Hirosawa, and K. Hono, J. Alloys Compd. 813, 152224 (2020).
[15] C. Nan-xian, H. Shi-qiang, W. Yu, and S. Jiang, J. Magn. Magn. Mater. 233, 169 (2001).

[16] T. Kuno, S. Suzuki, K. Urushibata, K. Kobayashi, N. Sakuma, M. Yano, A. Kato, and A. Manabe, AIP Adv. 6, 025221 (2016).

[17] I. Dirba, H. Sepehri-Amin, T. Ohkubo, and K. Hono, Acta Mater. 165, 373 (2019).

[18] P. Tozman, H. Sepehri-Amin, Y. K. Takahashi, S. Hirosawa, and K. Hono, Acta Mater. 153, 354 (2018).

[19] S. K. Makineni, M. Lenz, P. Kontis, Z. Li, A. Kumar, P. J. Felfer, S. Neumeier, M. Herbig, E. Spiecker, D. Raabe, and B. Gault, JOM 70, 1736 (2018).

[20] D. Palanisamy, D. Raabe, and B. Gault, Acta Mater. 174, 227 (2019).

[21] P. J. Felfer, T. Alam, S. P. Ringer, and J. M. Cairney, Microsc. Res. Technol. 75, 484 (2012).

[22] M. Herbig, P. Choi, and D. Raabe, Ultramicroscopy 153, 32 (2015).

[23] J. L. Murray, Bull. Alloy Phase Diagrams 2, 320 (1981).

[24] See Supplemental Material at http://link.aps.org/supplemental/ 10.1103/PhysRevMaterials.4.054404 for Change in Magnetic Domains of SmFeTi Based Permanent Magnet on Application of Magnetic Field.

[25] Yu. G. Pastushenkov, J. Magn. Magn. Mater. 140, 1063 (1995).

[26] Yu. G. Pastushenkov, O. B. Dyogteva, A. W. Shipov, and K. P. Skokov, J. Magn. Magn. Mater. 157, 67 (1996).

[27] X. Zhang, B. Cheng, and Y. Yang, Appl. Phys. Lett. 77, 4022 (2000).

[28] S. Huang and T. Chin, J. Appl. Phys. 70, 4439 (1991).

[29] C. Koestler, L. Schulz, and G. Thomas, J. Appl. Phys. 67, 2532 (1990). 
[30] F. Maccari, L. Schäfer, I. Radulov, L. V. B. Diop, S. Ener, E. Bruder, K. Skokov, and O. Gutfleisch, Acta Mater. 180, 15 (2019).

[31] J. Liu, H. Sepehri-Amin, T. Ohkubo, K. Hioki, A. Hattori, T. Schrefl, and K. Hono, Acta Mater. 82, 336 (2015).

[32] R. Ramesh, G. Thomas, and B. M. Ma, J. Appl. Phys. 64, 6416 (1988).

[33] R. Ramesh and K. Srikrishna, J. Appl. Phys. 64, 6406 (1988).

[34] J. Bernardi, J. Fidler, M. Sagawa, and Y. Hirose, J. Appl. Phys. 83, 6396 (1998).

[35] L. Schultz, K. Schnitzke, and J. Wecker, Appl. Phys. Lett. 56, 868 (1990).

[36] E. W. Singleton, J. Strzeszewski, and G. C. Hadjipanayis, Appl. Phys. Lett. 54, 1934 (1989).

[37] Y. B. Kim, K. S. Ryu, C. S. Kim, S. Sugimoto, M. Okada, and M. Homma, Mater. Trans. JIM 33, 407 (1992).

[38] J. Ding and M. Rosenberg, J. Magn. Magn. Mater. 80, 105 (1989).

[39] M. S. Anagnostou and D. Niarchos, J. Magn. Magn. Mater. 88, 100 (1990)

[40] S. H. Huang, T. S. Chin, Y. S. Chen, S. K. Chen, C. H. Lin, and P. Y. Lee, IEEE Trans. Magn. 26, 1391 (1990).
[41] H. Pan, F. Yang, C. Chen, N. Tang, X. Han, J. Wang, J. Hu, K. Zhou, R. Zhao, and Q. Wang, Solid State Commun. 98, 259 (1996).

[42] F.-M. Yang, B. Nasunjilegal, H.-Y. Pan, J.-L. Wang, R.-W. Zhao, B.-P. Hu, Y.-Z. Wang, H.-S. Li, and J. M. Cadogan, J. Magn. Magn. Mater. 135, 298 (1994).

[43] T. B. Martins and H. R. Rechenberg, Hyperfine Interact. 169, 1273 (2006).

[44] H. Samata, N. Fujiwara, Y. Nagata, T. Uchida, and M. Der Lan, J. Magn. Magn. Mater. 195, 376 (1999).

[45] W. Körner, G. Krugel, and C. Elsässer, Sci. Rep. 6, 24686 (2016).

[46] Tsung-Shune Chin, Cheng-Hao Lin, H. J. Bai, Y. H. Huang, K. S. Cheng, and S. H. Huang, IEEE Trans. Magn. 28, 2587 (1992).

[47] H. Sepehri-Amin, T. Ohkubo, T. Nishiuchi, S. Hirosawa, and K. Hono, Scr. Mater. 63, 1124 (2010).

[48] J. Liu, H. Sepehri-Amin, T. Ohkubo, K. Hioki, A. Hattori, T. Schrefl, and K. Hono, Acta Mater. 61, 5387 (2013).

[49] P. Tozman, Y. K. Takahashi, H. Sepehri-Amin, D Ogawa, S. Hirosawa, and K. Hono, Acta Mater. 178, 114 (2019). 